\title{
La aplicación de otros métodos de valoración en el régimen de precios de transferencia ${ }^{(*)(*)}$
}

\section{The application of alternative valuation methods under the transfer pricing regime}

\author{
Fernando Loayza Jordán ${ }^{(* *)}$ \\ Pontificia Universidad Católica del Perú
}

Martín La Rosa Almenara ${ }^{(* * *)}$

Universidad Peruana de Ciencias Aplicadas

\begin{abstract}
Resumen: Previamente a la última reforma del régimen peruano de precios de transferencia, solo se permitía la aplicación de métodos específicos de valoración, lo que generó una serie de problemas de índole práctica y constitucional. Estos se resolvieron parcialmente con la reciente inclusión de una disposición que permite la aplicación de "otros métodos". El presente trabajo analiza la problemática de la situación previa, así como las soluciones que presenta la reforma y sus límites. Por último, se propone una serie de precisiones que, a juicio de los autores, deberían incluirse en la reglamentación de los "otros métodos".
\end{abstract}

Palabras clave: precios de transferencia - Impuesto a la Renta - métodos de valoración - otros métodos - OCDE

Abstract: Prior to the last reform of the transfer pricing regime, only the application of specific valuation methods was allowed, which generated several practical and constitutional problematic issues. These were partially

$\left(^{*}\right) \quad$ Nota del Editor: este artículo fue recibido el 07 de octubre del 2017 y su publicación fue aprobada el 11 de noviembre del 2017.

${ }^{* *}$ Los autores agradecen los valiosos comentarios de Humberto Allemant, Miguel Puga y Rafael Urbaneja al presente trabajo y la asistencia de Ivo García Shapiama, Bachiller de Derecho por la Universidad Nacional Mayor de San Marcos

${ }^{* * *}$ Abogado por la Pontificia Universidad Católica del Perú. Estudios en la Facultad de Derecho y en la Facultad de Economía de la Universidad Pompeu Fabra, España. Estudios de especialización en Tributación Internacional y Precios de Transferencia en la Universidad de Leiden, Holanda. Asociado extraordinario de la Asociación Civil IUS ET VERITAS. Correo electrónico: fernando.loayza@pe.pwc.com

${ }^{(* * *}$ ) Bachiller de Derecho por la Universidad Peruana de Ciencias Aplicadas. Asociado del Estudio Rebaza, Alcázar \& De Las Casas. Correo electrónico: martin.larosa@rebaza-alcazar.com 
La aplicación de otros métodos de valoración en el régimen de precios de transferencia The application of alternative valuation methods under the transfer pricing regime

solved with the recent inclusion of a provision that allows the application of "other methods". This paper analyzes the issues of the previous situation, as well as the solutions presented by the reform and its corresponding limits. Finally, the authors propose several precisions that, in their opinion, should be included in the regulation of the "other methods".

Keywords: transfer pricing - corporate income tax - valuation methods - other methods - OECD

Sumario: 1. Introducción y planteamiento del problema_2. Antes de la reforma_2.1. ¿Supuesto regulado, laguna del derecho o vacío legal?_ 2.2. Las disposiciones que "fuerzan" métodos resultan inconstitucionales_3. La reforma del Decreto Legislativo No. 1312 y la aplicación de otros métodos_3.1. ¿Existe una jerarquía entre los métodos específicamente establecidos y los otros métodos?_3.2. ¿Qué puede regular el Reglamento de la Ley del IR respecto a los "otros métodos"?_4. Conclusiones_5. Referencias bibliográficas.

\section{Introducción y planteamiento del problema}

El régimen de precios de transferencia contenido en la Ley del Impuesto a la Renta peruano y en sus disposiciones reglamentarias (en adelante, "nuestro Régimen" o "el Régimen") establece una metodología particular para determinar el valor de mercado, a efectos del Impuesto a la Renta, (en adelante, "IR") de transacciones realizadas entre partes vinculadas o de aquellas que se realicen desde, hacia o a través de países o territorios de baja o nula imposición. En estos casos, no se determina la base imponible de las transacciones aplicando las reglas generales de valor de mercado establecidas en el artículo 32 de la Ley del IR, sino siguiendo el arm's length principle (en adelante, "ALP"), las disposiciones establecidas en el artículo 32-A de la Ley del IR y sus disposiciones reglamentarias.

Esta metodología tiene la particularidad de no determinar un monto exacto con el que se asigne el valor de una transacción. El Régimen, antes que establecer un valor exacto, regula un estándar ${ }^{(1)}$ para determinar la base imponible, dentro del cual podrán existir varios valores aceptados según el ALP.

Este estándar prescribe, en términos sencillos, que la base imponible del IR aplicable a una transacción se determinará por el valor que hubieran asignado partes independientes a transacciones comparables, en condiciones iguales o similares. En consecuencia, el precio convenido por las partes será irrelevante para las transacciones sujetas al Régimen, el cual cuantificará la base imponible de éstas siguiendo sus disposiciones particulares. Tales disposiciones establecen los métodos, análisis y directrices para determinar el rango de valores del ALP.

Hasta el ejercicio 2016, nuestro Régimen solo contemplaba algunos métodos específicos para determinar el ALP de una transacción. Éstos eran los métodos de valoración que la OCDE incluía expresamente en sus Directrices (OECD Transfer Pricing Guidelines for Multinational Enterprises and Tax Administrations, en adelante "Directrices OCDE")(2). Sin embargo, a diferencia de las Directrices OCDE, que si

(1) Es importante recordar que, en tanto el ALP es un estándar, no existe una valorización única de una transacción, sino un rango de valores aceptables según el ALP. 'La valoración 'a mercado' de las operaciones vinculadas no es un concepto unívoco: no existe un único valor 'de mercado', sino en todo caso un rango de valdo seleccionado ofrece una solución más satisfactoria (...)". Véase Ramón López de Haro Estejo, Mario Ortega Calle y Rafael Cabello Gil, "Los precios de transferencia: Normativa y Tendencias" en Fiscalidad Internacional (1) 5ta edición. Director: Fernando Serrano Antón. (Centro de Estudios Financieros, 2012), 572-3.

(2) En efecto, el Capítulo II de las Directrices OCDE reconoce dos clases de métodos: (i) los métodos tradicionales basados en las operaciones (traditional transaction methods); y, (ii) los métodos basados en los resultados de las operaciones (transactional profit methods). Estas son las mismas clases de métodos que reconoce nuestro Régimen, aunque cabe señalar que a diferencia de las Directrices OCDE, que tratan al profit split method ("método de la distribución del resultado") como un solo método, nuestro Régimen lo trata como dos métodos diferenciados: (i) el método de la partición de utilidades; y, (ii) el método residual de partición de utilidades, aunque ambos son modalidades del profit split method. 


\section{Fernando Loayza Jordán y Martín La Rosa Almenara}

contemplan claramente la posibilidad de aplicar métodos no especificados en las mismas ${ }^{(3)}$, los métodos de valoración parecían estar enumerados de forma taxativa.

Esto generaba un problema con aquellas transacciones cuyas características no permitían la aplicación apropiada de uno de los métodos. Como establece el inciso e) del artículo 32-A de la Ley, al elegir el método de valoración aplicable se debe tomar en cuenta cuál es la mejor forma de aproximarnos a la realidad económica de una transacción:

"Los precios de las transacciones sujetas al ámbito de aplicación de este artículo serán determinados conforme a cualquiera de los siguientes métodos internacionalmente aceptados para cuyo efecto deberá considerarse el que resulte más apropiado para reflejar la realidad económica de la operación" (Énfasis agregado).

Sin embargo, el hecho que nuestro Régimen obligara a los contribuyentes a elegir entre una lista taxativa de métodos de valoración nos enfrentaba a una complicada disyuntiva. De facto, los contribuyentes se veían obligados a forzar los métodos a disposición (por ejemplo, utilizando comparables inadecuados o aplicando métodos sin la información necesaria para aplicarlos adecuadamente). Esto implicaba una clara contradicción con el ALP, pues forzar un método de valoración solía resultar en un rango de valor de mercado distorsionado y ajeno al ALP. Además, al tratarse de una aplicación forzada, el análisis de precios de transferencia resultaba extremadamente endeble y susceptible de cuestionamientos por parte de la Administración Tributaria.

Para solucionar este problema, el Decreto Legislativo No. 1312 introdujo el numeral 7 al inciso e) del artículo 32-A de la Ley del IR. Con esta modificación (en adelante, "la reforma"), desde el 01 de enero de 2017, nuestra legislación permite la aplicación de métodos de valoración distintos a los que se encontraban específicamente establecidos en la Ley:

"Artículo 32-A.- En la determinación del valor de mercado de las transacciones a

(3) "2.9. Además, los grupos multinacionales conservan la libertad de aplicar métodos no descritos en estas Directrices (denominados en lo sucesivo "otros métodos") para determinar sus precios, siempre que estos satisfagan el principio de plena competencia en los términos descritos en estas Directrices. Sin embargo, estos otros métodos no deben usarse como sustitutivos de los métodos reconocidos por la OCDE cuando estos últimos resulten más apropiados en función de los hechos y circunstancias del caso. Cuando se recurra a los otros métodos, su elección debe estar fundamentada en una explicación de por qué se considera que los métodos reconocidos por la OCDE se consideran menos apropiados o inviables en unas circunstancias concretas, y la razón de por qué se considera que el otro método seleccionado ofrece una solución más satisfactoria (...)" (énfasis agregado).

Cabe señalar que, si bien el inciso h) del artículo 32-A de la Ley señala que: "Para la interpretación de lo dispuesto en este artículo, serán de aplicación las Guías sobre Precios de Transferencia para Empresas Multinacionales y Administraciones Fiscales, aprobadas por el Consejo de la Organización para la Cooperación y el Desarrollo Económico-OCDE, en tanto las mismas no se opongan a las disposiciones aprobadas por esta Ley", en nuestra opinión esta remisión no permite que vía interpretación se permita la aplicación de otros métodos. La aceptación de otros métodos por esta vía vulneraría el principio de reserva de ley tributaria, pues las disposiciones del Régimen son normas de base imponible, toda vez que terminan determinan el valor respecto al que se gravara una transacción. Esta opinión coincide con la de Villanueva Gutiérrez que señala que el Régimen es una norma de "valoración de la base imponible del IR que debe ser aplicada por los contribuyentes en la autoliquidación del IR" y con la de Gamba Valega, cuando señala que "las reglas contenidas en los artículos 32 y 32-A de la LIR obligan a los contribuyentes a valorizar sus operaciones con partes vinculadas y con sujetos residentes en paraísos fiscales, aplicando el principio de libre concurrencia. Por tanto, se tratan de normas que regulan la base imponible del IR en determinadas circunstancias, con total independencia de si la Administración Tributaria efectuó o no algún ajuste". Al respecto véase, respectivamente a Walker Villanueva Gutierrez, "Precios de Transferencia en el IGV," Vectigalia 3 (Año 3 2007): 110; y a César Gamba Valega, "Algunos aspectos constitucionales sobre el régimen de precios de transferencia," PT - Precios de Transferencia IFA-Perú (Junio 2008): 239. 
que se refiere el numeral 4) del artículo 32, deberá tenerse en cuenta las siguientes disposiciones

$(\ldots)$

e) Métodos utilizados

Los precios de las transacciones sujetas al ámbito de aplicación de este artículo serán determinados conforme a cualquiera de los siguientes métodos internacionalmente aceptados, para cuyo efecto deberá considerarse el que resulte más apropiado para reflejar la realidad económica de la operación:

$(\ldots)$

\section{7) Otros métodos}

Cuando por la naturaleza y características de las actividades y transacciones no resulte apropiada la aplicación de ninguno de los métodos anteriores, podrá acudirse a la aplicación de otros métodos, de acuerdo a lo que establezca el reglamento" (Énfasis agregado).

\section{Antes de la reforma}

Antiguamente, las limitaciones de los métodos de valoración podían resolverse acudiendo a las normas generales de valor de mercado. En efecto la Ley 27024, publicada en el año 1998, que modificó el Decreto Legislativo No. 774 (la antigua Ley del Impuesto a la Renta) e introdujo de forma incipiente el ALP, permitía acudir a las normas generales de valor de mercado en caso el rango del ALP no pudiera determinarse:

"4. Para las transacciones entre empresas vinculadas económicamente, el que normalmente se obtiene en las operaciones que la empresa realiza con terceros no vinculados, en condiciones iguales o similares; debiendo tenerse en cuenta todos los elementos que sean pertinentes. De no poder determinarse este valor, será el que establezca el mercado" (Énfasis agregado).

Así, hasta su derogación, esta versión del artículo 32 había previsto una solución al problema planteado: si la determinación del ALP para una transacción no era posible, el contribuyente podía valorizar tal transacción a valor de mercado. Sin embargo, con la publicación del Decreto Legislativo No. 945 (vigente desde el ejercicio 2004), se modificó la estructura del artículo
32, introduciendo así el artículo 32-A y, en gran medida, el modelo aceptado internacionalmente según los lineamientos de la OCDE.

Con esta reforma se eliminó la posibilidad de acudir a las normas generales de valor de mercado, presentándose el problema que nos ocupa: ¿Cómo enfrentar los casos en los que la aplicación de alguno de los métodos de valoración existentes sea insuficiente debido a las características específicas de las transacciones bajo análisis? En este punto, consideramos necesario detenernos a evaluar si desde la entrada en vigencia del Decreto Legislativo No. 945 hasta la reforma (es decir, desde el ejercicio 2004 hasta el ejercicio 2016) nos encontrábamos ante una laguna o ante un vacío legal en aquellos casos en los que los métodos a disposición eran insuficientes y el Régimen nos obligaba a forzarlos.

2.1. ¿Supuesto regulado, laguna del derecho o vacío legal?

Como expondremos brevemente, sólo podrá analizarse si nos encontramos ante una laguna del derecho o ante un vacío legal, cuando nos encontramos ante un supuesto no regulado. No bastará que un supuesto esté regulado de forma deficiente para considerarlo como un supuesto no regulado.

En términos de Marcial Rubio,

"la laguna del Derecho puede ser definida como aquel suceso para el que no existe norma jurídica aplicable, pero que se considera que debiera estar regulado por el sistema jurídico. Estrictamente hablando, el suceso que da origen a la laguna no está previsto en ninguno de los supuestos existentes en las normas vigentes del sistema jurídico, o puede ocurrir también que, a la consecuencia prevista, deba añadirse otra no prevista para el mismo supuesto" (Rubio 2012, 261) (Énfasis agregado). 


\section{Fernando Loayza Jordán y Martín La Rosa Almenara}

El mismo autor señala que, "existe un concepto teórico que, por contraste con el de laguna, (...); es el vacío del Derecho entendiendo por tal un suceso para el que no existe norma jurídica aplicable y que se considera que no debe estar regulado por el Derecho (...)" (Rubio 2012, 261) (Énfasis agregado).

Por lo tanto, debemos dilucidar si existía un supuesto no regulado en nuestro Régimen antes de determinar si tal supuesto no regulado configuraría una laguna o un vacío jurídico. En nuestra opinión, tal análisis debe partir del enunciado lingüístico de la disposición en cuestión. El inciso e) del artículo 32-A de la Ley del IR establece que "Los precios de las transacciones sujetas al ámbito de aplicación de este artículo serán determinados conforme a cualquiera de los siguientes métodos internacionalmente aceptados (...)" (Énfasis agregado).

Como vemos se señala expresamente que la valorización de las transacciones sujetas al Régimen se determinara únicamente conforme a los métodos expresamente recogidos en el inciso e). Al parecer, nuestra Ley del IR consideraba que los seis métodos expresamente recogidos eran aplicables para cualquier transacción que se encuentre en el ámbito de aplicación del Régimen. De esta manera, no contemplaba que alguna transacción no pudiera valorizarse con los métodos específicamente recogidos por el Régimen.

En consecuencia, consideramos que no nos encontramos ante un supuesto de hecho no regulado, sino más bien ante un supuesto de hecho regulado deficientemente. ¿Esto implica que no tenemos más opción que aplicar los métodos disponibles de forma "forzada"? No. Según nuestro análisis, consideramos que desde un punto de vista constitucional tributario, el "forzar" los métodos sería inaceptable, por las razones que expondremos a continuación.

\subsection{Las disposiciones que fuerzan métodos resultan inconstitucionales}

En nuestra opinión, la obligación que imponía el Régimen antes de la reforma de "forzar" los métodos estipulados taxativamente a todas las transacciones en las que el Régimen fuera aplicable implica una vulneración a los principios constitucionales tributarios de igualdad, capacidad contributiva y no confiscatoriedad.

Como sabemos, el principio de igualdad no implica una prohibición de toda distinción de trato jurídico, sino que defiende que "las situaciones económicas iguales deben ser tratadas de la misma manera y, a la inversa, situaciones económicamente disímiles, deben recibir un trato diferenciado" (Landa 2006 , 44). Por lo tanto, se habría estado vulnerando el principio de igualdad si en aplicación del Régimen se hubieran utilizado los mismos métodos para valorizar todas las transacciones, aunque estas tuvieran una naturaleza distinta, que impidiera que la valorización resulte conforme a la realidad económica de tales transacciones $y$ al ALP.

Por otro lado, el forzar los métodos estipulados puede conllevar a valorizar las transacciones de forma poco idónea, sin tomar en cuenta su verdadera realidad económica. Por ello, vulneraría el principio de capacidad contributiva, que obliga al legislador a considerar la capacidad económica de un sujeto al momento de establecer las obligaciones tributarias del mismo (Fernández Cartagena 2006, 172) y el principio de no confiscatoriedad, que evita que las normas tributarias puedan afectar gravemente la esfera patrimonial de los particulares en casos concretos (Danós 1994, 139) y "cumple un rol de refuerzo de la garantía de la propiedad como límite al poder impositivo estatal" (Danós 2006, 141).

(4) Nótese la contradicción que existía en el mismo párrafo del artículo 32-A cuando se exigía que los precios de las transacciones se determinen conforme a uno de los métodos específicamente establecidos en el Régimen, pero al mismo se exigía que el elegido resulte "apropiado para reflejar la realidad económica de la operación", aun cuando los mencionados métodos no resultaran los más apropiados para reflejar tal realidad económica. 
La aplicación de otros métodos de valoración en el régimen de precios de transferencia The application of alternative valuation methods under the transfer pricing regime

En consecuencia, una disposición tributaria que obligue a determinar el IR sobre una base imponible determinada sin tomar en cuenta la realidad económica de la operación no sólo iría en contra de la lógica del Régimen y de disposiciones concretas de la Ley del IR ${ }^{(4)}$ sino que también parecería sufrir de graves vicios de inconstitucionalidad.

La imposibilidad de cuestionar la constitucionalidad de esta disposición en sede administrativa, dada la prohibición de aplicar control difuso administrativo(5), aparentemente evitaría que la Administración Tributaria o el Tribunal Fiscal pudieran aceptar métodos no contemplados en el Régimen vigente hasta el 2016. Sin embargo, consideramos que existen dos caminos posibles para los contribuyentes que decidan defender la aplicación de otros métodos durante este periodo:

- Proceder por la vía de una acción de amparo(6).

- Solicitar que se aplique control de convencionalidad en sede administrativa(7).

\section{La reforma del Decreto Legislativo No. 1312 y la aplicación de otros métodos}

Con la introducción de la reforma y la posibilidad de aplicar otros métodos de valoración, el problema que venimos analizando parecería resolverse: en aquellos casos en los que los métodos específicamente contemplados en el Régimen sean insuficientes o su aplicación se torne "forzada", podrán aplicarse otros métodos de valoración. Entre los casos más usuales nos encontramos con transacciones vinculadas a activos intangibles, acciones de empresas que no cotizan en bolsa, contratos atípicos, entre otros.

Sin embargo, esto no quiere decir que en estos casos se permitirá el uso de cualquier método de valoración. Debemos recordar que aún con la reforma, la piedra angular de

(5) Recordemos que el Tribunal Constitucional en la sentencia recaída en el Expediente $N^{\circ}$ 04293-2012-AA/TC dejo sin efecto "el precedente vinculante contenido en la STC 03741-2004-PA/TC, conforme al cual se autorizaba a todo tribunal u órgano colegiado de la Administración Pública a inaplicar una disposición infraconstitucional cuando considere que ella vulnera manifiestamente la Constitución, sea por la forma o por el fondo“. Este nuevo criterio impide que la Administración Tributaria y el Tribunal Fiscal puedan inaplicar una norma tributaria, por más que se compruebe su manifiesta inconstitucionalidad.

(6) De acuerdo al artículo 37 del Código Procesal Constitucional "el amparo procede en defensa de los siguientes derechos: 1) de igualdad y de no ser discriminado por razón de origen, sexo, raza, orientación sexual, religión, opinión, condición económica, social, idioma, o de cualquier otra índole;(...) 12) De propiedad y herencia; (... 25) Los demás que la Constitución reconoce." Sin embargo, sería necesario evaluar si en el caso concreto se podría aplicar alguna de las excepciones al requisito de agotamiento de las vías previas (artículo 46 del Código Procesal Constitucional).

(7) El control de convencionalidad es "aquel ejercicio que consiste en verificar la compatibilidad de las normas y demás prácticas internas con la Convención Americana sobre Derechos Humanos; la jurisprudencia de la Corte Interamericana de Derechos Humanos y los demás tratados interamericanos de los cuales el Estado sea parte". Al respecto, véase Elard Bolaños, “¿Puede el Control de Convencionalidad ser una Alternativa ante la prohibición del Control Difuso en sede Administrativa?" Administración Pública \& Control 27 (marzo 2016): 66. Cabe señalar que la Corte Interamericana de Derechos Humanos $(\mathrm{CIDH})$ ha especificado que el control de convencionalidad no solo debe aplicarse en sede judicial, sino también en sede administrativa. A modo de ejemplo, podemos citar el caso "Personas Dominicanas y Haitianas expulsadas vs República Dominicana" en el que la CIDH estableció que: "471. (...) esta Corte considera pertinente recordar, sin perjuicio de lo ordenado, que en el ámbito de sus competencias "todas las autoridades y órganos de un Estado Parte en la Convención tienen la obligación de ejercer un control de convencionalidad". En nuestra opinión, en este caso particular, el control de convencionalidad debería sustentarse en el derecho de igualdad ante la ley y el derecho a la propiedad privada, contempladas expresamente en la Convención Americana sobre Derechos Humanos. Al respecto, véase el caso Cantos vs Argentina Sentencia de 28 de noviembre de 2002 (Fondo, Reparaciones y Costas). 


\section{Fernando Loayza Jordán y Martín La Rosa Almenara}

nuestro Régimen sigue siendo el ALP y, por tanto, cualquier otro método empleado deberá seguir el razonamiento del tal principio. En línea con lo mencionado, métodos inspirados en aproximaciones contrarias al ALP, como el global formulary apportionment ${ }^{(8)}$ no están permitidos.

Así también lo especifican las Directrices OCDE:

"2.9. Además, los grupos multinacionales conservan la libertad de aplicar métodos no descritos en estas Directrices (denominados en lo sucesivo "otros métodos") para determinar sus precios, siempre que estos satisfagan el principio de plena competencia en los términos descritos en estas Directrices (...)" (énfasis agregado).

Por su parte, aquellos métodos de valorización financiera de aplicación usual en el mercado deben permitirse, toda vez que son los métodos que se utilizan para valorizar las transacciones realizadas entre terceros independientes. Así, cualquier método de valorización que utilicen partes independientes de una transacción a efectos de establecer su precio, como los métodos de descuento de flujos ${ }^{(9)}$, deberían permitirse. Debemos recordar que, finalmente, la intención del ALP es simular las condiciones normales de mercado.

Dicho esto, nos interesa analizar dos potenciales problemas que presenta la forma en la que se ha establecido legislativamente la posibilidad de aplicar otros métodos: (i) la aparente jerarquía que establece el nuevo numeral 7 entre los métodos específicamente establecidos y otros métodos y (ii) la posibilidad, que parece permitir el enunciado del numeral 7 , de regular los otros métodos aplicables por vía reglamentaria.

\section{1. ¿Existe una jerarquía entre los métodos específicamente establecidos y los otros métodos?}

La forma en la que el nuevo numeral 7 regula a los "otros métodos" parece dar a entender que estos están en una jerarquía inferior a los métodos específicamente aceptados: parecería que solo se podría acudir a "otros métodos" como una especie de ultima ratio. Aparentemente, se estaría siguiendo la misma línea que las Directrices OCDE, cuando señalan que existe una carga de la prueba particular para la aplicación de "otros métodos":

"2.9. (...) Sin embargo, estos otros métodos no deben usarse como sustitutivos de los métodos reconocidos por la OCDE cuando estos últimos resulten más apropiados en función de los hechos y circunstancias del caso. Cuando se recurra a los otros métodos, su elección debe estar fundamentada en una explicación de por qué se considera que los métodos reconocidos por la OCDE se consideran menos apropiados o inviables en unas circunstancias concretas, y la razón de por qué se considera que el otro método seleccionado ofrece una solución más satisfactoria (...)" (énfasis agregado).

A nuestro parecer, la forma en la que el nuevo numeral 7 establece la carga de la prueba particular para la aplicación de otros métodos

(8) A modo de síntesis, podemos señalar que la principal diferencia entre el ALP y el GFA es que el ALP parte de tratar cada parte de un grupo empresarial como contribuyentes individuales, que hipotéticamente deberían realizar sus transacciones a valor de mercado. Por otro lado, el GFA parte de tratar a todo el grupo empresarial como una unidad, para luego proceder a determinar cuánto corresponde a cada parte de tal unidad. Al respecto, véase Reuven S. Avi-Yonah, "The Rise and Fall of Arm's Length: A Study in the Evolution of U.S. International Taxation," U of Michigan Law \& Economics, Olin Working Paper No. 07-017 o U of Michigan Public Law Working Paper No. 92 (September 27, 2007); p.3.

(9) En términos de Pablo Fernández, "los métodos de descuento de flujos se basan en el pronóstico detallado y cuidadoso, para cada período, de cada una de las partidas financieras vinculadas a la generación de los cash flows correspondientes a las operaciones de la empresa, como por ejemplo, el cobro de ventas, los pagos de mano de obra, de materias primas, administrativos, de ventas, etc., y la devolución de créditos, entre otros." Pablo Fernández, "Métodos de valoración de empresas," (12 de febrero de 2016). IESE: Universidad de Navarra, SSRN: https://ssrn.com/abstract=1267987 
La aplicación de otros métodos de valoración en el régimen de precios de transferencia The application of alternative valuation methods under the transfer pricing regime

parte de la cuestionable premisa que la aplicación de un método puede resultar apropiada o no a un caso concreto:

\section{"7) Otros métodos}

Cuando por la naturaleza y características de las actividades y transacciones no resulte apropiada la aplicación de ninguno de los métodos anteriores, podrá acudirse a la aplicación de otros métodos, de acuerdo a lo que establezca el reglamento" (énfasis agregado).

Esta forma binaria de analizar la aplicabilidad de un método nos parece imprecisa porque la práctica nos demuestra que la selección de un método de valorización pasa por un proceso de análisis de distintas variables que ponderar y no de una suerte de checklist que pueda cumplirse o no. La elección de un método de valorización no es una ciencia exacta que resulta en la elección de un solo método aplicable.

En este punto, las Directrices OCDE son más atinadas, pues utilizan los términos más apropiados o menos apropiados. En efecto, un método de valorización casi siempre podrá, en estricto, aplicarse. Siempre se puede buscar comparables más lejanas, utilizar información más limitada (o estimaciones menos certeras), realizar un análisis funcional más laxo, entre otros. Por lo tanto, casi siempre podrán aplicarse todos los métodos explícitamente recogidos por el Régimen a un caso concreto. De hecho, como analizamos en el acápite anterior, esto es lo que se hacía hasta el 2016: forzar los métodos explícitamente contemplados en el Régimen.

En consecuencia, establecer como un requisito para aplicar "otros métodos" que se descarte completamente la posibilidad de aplicar los métodos adoptados explícitamente en el Régimen implicaría que sería casi imposible aplicar estos "otros métodos".

Por ello, en nuestra opinión, debemos aplicar un criterio similar al que se llegó tras el antiguo debate entre los traditional transaction methods y los transactional profit methods: no es necesario comprobar la imposibilidad de un método especificado para poder aplicar otro método, basta demostrar que éste último refleja mejor la realidad económica de la transacción. En consecuencia, no existirá una mayor carga de la prueba para aplicar otros métodos que para aplicar uno de los métodos explícitamente contemplados en el Régimen.
En cualquier caso, el contribuyente siempre deberá demostrar por qué el método elegido refleja mejor la realidad económica de la transacción bajo análisis.

\section{2. ¿Qué puede regular el Reglamento de la} Ley del IR respecto a los "otros métodos"? Es llamativo que el nuevo numeral 7 permita acudir a la aplicación de otros métodos, "de acuerdo a lo que establezca el reglamento", toda vez que, como hemos señalado, los métodos de valorización son normas de base imponible protegidas por el principio de reserva de ley. En esa línea, el Reglamento de la Ley del IR no debería establecer qué nuevos métodos serían aplicables o no (más allá de una posible referencia a la imposibilidad de aplicar métodos incompatibles con el arm's length principle, que no sería más que el desarrollo reglamentario de una delimitación legal), pues dicho desarrollo le corresponde a una norma con rango de ley.

Por lo tanto, no podemos interpretar que la indicación "de acuerdo a lo que establezca el reglamento" implique que la aplicación de otros métodos se encuentre supeditada a la entrada en vigencia de una norma reglamentaria. No será necesario que el Reglamento de la Ley del IR adopte otros métodos de forma específica para poder aplicarlos y, más aún, esta adopción específica podría generar problemas de reserva de ley.

No obstante, el Reglamento sí podría (y nos atrevemos a indicar que debería) incluir disposiciones que faciliten la aplicación de otros métodos. A modo de ejemplo, podemos citar las siguientes:

a) Precisar que las únicas limitaciones a la aplicación de "otros métodos" son (i) su concordancia con el arm's length principle y (ii) que reflejen de la forma más idónea la realidad económica de la transacción bajo análisis. 


\section{Fernando Loayza Jordán y Martín La Rosa Almenara}

b) Señalar, a modo de ejemplo, algunos de los "otros métodos" aplicables (por ejemplo, las tasaciones).

c) Tal como hace el artículo 113 del Reglamento de la Ley del IR, se podrían incluir casos en los que los métodos específicos no resulten recomendables, a fin de establecer directrices para facilitar que los contribuyentes puedan identificar aquellos casos en los que deberían contemplar la aplicación de "otros métodos".

d) Precisar cuándo una versión modificada de un método específico pasa a ser "otro método" y se libera de los requisitos o características particulares que establece la Ley y el Reglamento para tal método específico.

Disposiciones que especifiquen que las normas generales aplicables a los métodos de valorización específicos también son aplicables a los "otros métodos", excepto en aquellos casos puntuales en los que las características del "otro método" no permitan su aplicación. Por ejemplo, no tendría sentido pretender aplicar la regulación de comparables a métodos que no se basen en la comparación con otras transacciones idénticas o similares.

Lamentablemente, las últimas modificaciones al Reglamento de la Ley del IR en materia de Precios de Transferencia ${ }^{(10)}$ no han incluido disposición alguna respecto a los "otros métodos", centrándose únicamente en las nuevas obligaciones formales exigidas por el Régimen desde su última reforma.

\section{Conclusiones}

Previo a la referida reforma, los contribuyentes se encontraban limitados a aplicar únicamente los métodos de valoración específicamente estipulados en el Régimen. Esto generaba que, en ocasiones, se forzaran tales métodos, pues existía la obligación de aplicar alguno de estos aunque no fueran los más idóneos debido a la realidad económica particular de ciertas transacciones. Desde nuestro punto de vista, forzar tales métodos no sólo era incongruente con el ALP, sino que también resultaba inconstitucional, pues vulneraba los principios constitucionales tributarios de igualdad, capacidad contributiva y no confiscatoriedad.
Con la última reforma del Régimen, se resuelve el mencionado problema, pues se permite acudir a otros métodos alternativos a los específicamente regulados en la Ley del IR. Estos otros métodos deberán seguir la lógica del ALP y aplicarse cuando sean los más indicados para reflejar la realidad económica de las transacciones bajo análisis. Si bien la norma parece indicar una subordinación de los otros métodos frente a los métodos específicos, en nuestra opinión establecer una jerarquía entre los métodos puede implicar que se aplique un método que no refleje de la forma más idónea la realidad económica de la transacción, contrariando la lógica del Régimen.

Por último, cualquier desarrollo reglamentario de los otros métodos deberá respetar el principio de reserva de ley. A nuestro entender, esto implica que el Reglamento no establezcan métodos específicos, sino que se limite a establecer directrices que faciliten la aplicación de otros métodos.

\section{Referencias bibliográficas}

Avi-Yonah, Reuven S. The Rise and Fall of Arm's Length: A Study in the Evolution of U.S. International Taxation. $U$ of Michigan Law \& Economics, Olin Working Paper No. 07-017 ○ $U$ of Michigan Public Law Working Paper No. 92 (September 27, 2007). http://dx.doi. org/10.2139/ssrn.1017524

Danós Ordóñez, Jorge E. 1994. El Régimen Tributario en la Constitución: Estudio Preliminar. Themis, No. 29, 139

Danós Ordóñez, Jorge. 2006. El principio constitucional de no confiscatoriedad de los tributos en la jurisprudencia del Tribunal Constitucional peruano. En: Temas de Derecho Tributario y de Derecho Público.

(10) Decreto Supremo No. 333-2017-EF. 
La aplicación de otros métodos de valoración en el régimen de precios de transferencia The application of alternative valuation methods under the transfer pricing regime

Libro Homenaje a Armando Zolezzi Moller. Lima: Palestra, 141.

Elard Bolaños, ¿Puede el Control de Convencionalidad ser una Alternativa ante la prohibición del Control Difuso en sede Administrativa? Administración Pública \& Control N²7 (marzo 2016).

Fernández Cartagena, Julio A. 2006. La capacidad contributiva. En: Temas de Derecho Tributario y de Derecho Público. Libro Homenaje a Armando Zolezzi Moller. Lima: Palestra, 172.

Fernández, Pablo. 2016. Métodos de valoración de empresas. IESE: Universidad de Navarra. http://dx.doi.org/10.2139/ssrn.1267987
Gamba Valega, César. 2008. Algunos aspectos constitucionales sobre el régimen de precios de transferencia. $P T$ - Precios de Transferencia. IFA-Perú, 239.

Landa Arroyo, César. 2006. Los principios tributarios en la Constitución de 1993. Una perspectiva constitucional. En: Temas de Derecho Tributario y de Derecho Público. Libro Homenaje a Armando Zolezzi Moller. Lima: Palestra

López de Haro Estejo, Ramón, Mario Ortega Calle y Rafael Cabello Gil. 2012. Los precios de transferencia: Normativa y Tendencias. Centro de Estudios Financieros, 572-573.

Rubio, Marcial. 2012. El Sistema Jurídico. Introducción al Derecho. Lima: Fondo Editorial de la Pontificia Universidad Católica del Perú
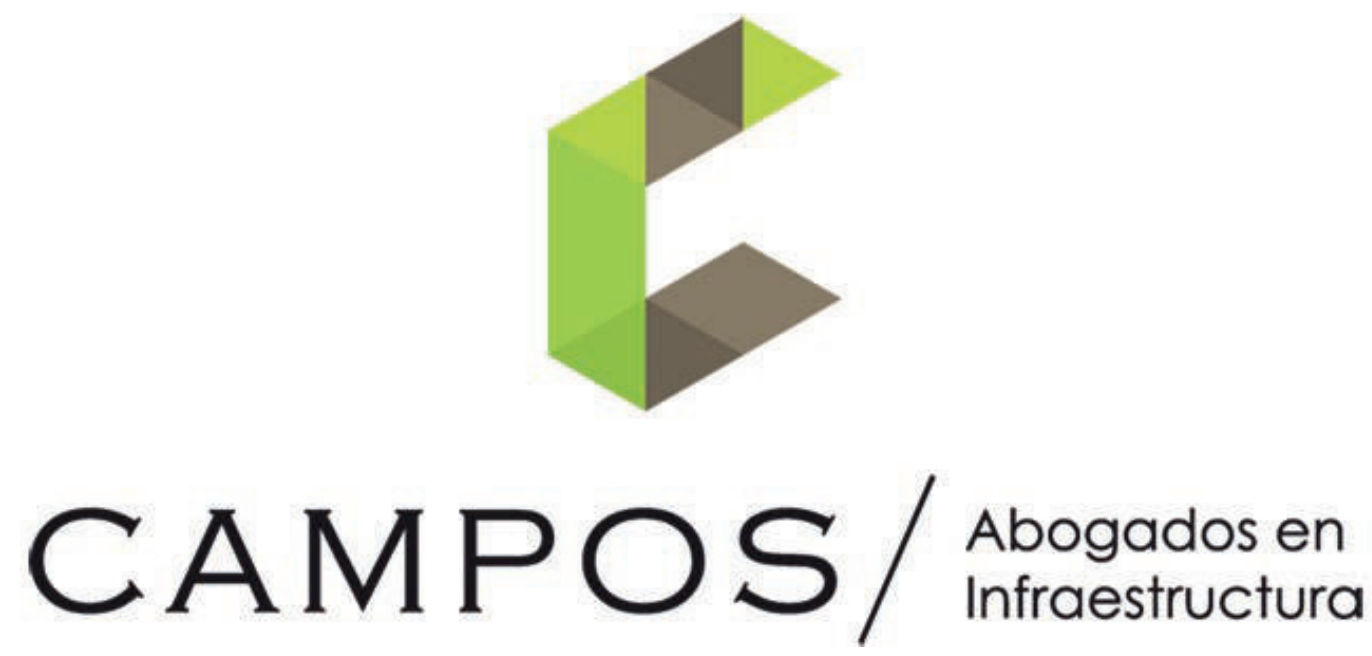